\title{
High-Grade Epstein-Barr Virus-Negative Biphenotypic Lymphoma with Expression of B- and T-Cell Markers and Leukemia Presentation: Case Report and Literature Review
}

\author{
Samah Kohla ${ }^{a}$ b $\quad$ Feryal A. Ibrahim ${ }^{a}$ Deena Mudawi ${ }^{c}$ Susanna Akiki ${ }^{d}$ \\ Dina Soliman $^{a}$ Ahmad Al-Sabbagh ${ }^{a}$ Reda R.H. Youssefe \\ Mohamed A. Yassinc \\ aDepartment of Lab Medicine and Pathology, Hematology Division, Hamad Medical \\ Corporation, Doha, Qatar; ${ }^{b}$ Department of Clinical Pathology, Hematology Division, \\ Faculty of Medicine, Al-Azhar University, Cairo, Egypt; 'Department of \\ Hematology-Oncology, Hamad Medical Corporation, Doha, Qatar; dDepartment of Lab \\ Medicine and Pathology, Molecular and Cytogenetic Division, Hamad Medical Corporation, \\ Doha, Qatar; ${ }^{e}$ Department of Clinical Imaging, Hamad Medical Corporation, Doha, Qatar
}

\section{Keywords}

Lymphoma - Biphenotypic lymphoma - Bigenotypic lymphoma - High-grade lymphoma .

CD3-positive B-cell lymphoma · Epstein-Barr virus-negative lymphoma

\begin{abstract}
Lymphomas are presently categorized according to their origin from B or T lymphocytes. The co-expression of CD3 in B-cell lymphomas or CD20 in T-cell lymphomas has been rarely reported. Immature and less often mature lymphomas may incorporate the rearrangements of both B- and T-cell antigen receptor genes (dual genotype or bigenotype). Lymphoma cells with a sole genotype hardly concurrently express both B- and T-cell markers (biphenotypic lymphomas). We describe a 63-year-old female who was presented with obstructive jaundice and epigastric pain of 10 days. Initial CBC revealed $43 \times 10^{3} / \mu \mathrm{L}$ white blood cells, $11.2 \mathrm{~g} / \mathrm{dL}$ hemoglobin, and $88 \times 10^{3} / \mu \mathrm{L}$ platelets. CT abdomen revealed hepatomegaly and suspected pancreatic mass with large retroperitoneal lymph nodal mass. Peripheral smear showed $56 \%$ lymphoid cells with blast morphology. The bone marrow (BM) aspirate smear was infiltrated by $83 \%$ immature-looking cells. BM biopsy showed interstitial to diffuse extensive infiltration by primitive-looking cells, positive for pan-B-cell antigens CD20, CD79, and PAX5 as well as the T-cell antigen CD4, CD5, CD3, while negative for all immaturity markers (CD34, TdT, and CD1a).
\end{abstract}


In situ hybridization for Epstein-Barr virus (EBV)-encoded small RNA (EBER) was negative. Flow cytometry on BM aspirate showed an abnormal population (50\%) expressing the B-cell antigens (CD19, CD20, CD79, CD22) and CD10, and showed lambda light chain restriction as well as the T-cell antigens CCD3 and CD4 with partial CD5. The analysis showed, also, another abnormal population of lambda restricted monotypic B cells (8\%) with dimmer CD45 (blast gate) and showed the same immunophenotype (expressing the B-cell antigens), but negative for CD10, cCD3, CD5, and CD4. Conventional cytogenetic revealed complex karyotype. Molecular studies revealed rearrangements of the immunoglobulin heavy chain region consistent with a clonal B-cell population. TCR gene rearrangement analysis was equivocal concerning clonality but was not conclusive for clonal T-cell disease. Our final diagnosis was peripheral blood and BM involvement by EBV-negative high-grade lymphoid neoplasm (in leukemic phase with blast morphology) and an ambiguous immunophenotype with a differential diagnosis that may include the rare entity of bigenotypic lymphoma or an unusual case of high-grade B-cell lymphoma with aberrant expression of T-cell markers (biphenotypic lymphomas).

\section{Introduction}

The diagnosis of lymphoma is based on histomorphology, immunophenotyping, and molecular studies. Lymphomas are broadly classified as being of the B-cell or T-cell lineages, using immunohistochemistry and/or flow cytometry. CD20 and CD3 are the most frequently used markers to determine B- and T-cell lineages. B-cell lymphomas with aberrant expression of T-cell-associated antigens such as CD2, CD4, CD8, CD5, CD7, and CD43 or T-cell lymphomas with aberrant expression of the B-cell marker CD20 have been reported [1]. However, cases of B-cell lymphoma with CD3 co-expression are extremely rare [2]. Immunophenotyping by flow cytometry and immunohistochemistry may be complemented by a molecular search for clonal rearrangements in B-cell receptors gene loci such as the immunoglobulin heavy (IGH) and light (IGK) chain genes and the T-cell receptor (TCR) gene loci gamma, delta, and beta. Increasingly common use of molecular tools has shown that simultaneous rearrangement of both IGH and TCR is not a rare event, especially in immature (lymphoblastic) cell populations. In lymphoma cases with a mixed population, simultaneous cross-lineage rearrangements have been documented to occur in about 10\% [3] or even more (35\%) in diffuse large B-cell lymphoma (DLBCL) group [4]. This may reflect either a bigenotypic lymphoma in which IGH and TCR rearrangements occur in the same cell or a composite lymphoma of so-called dual genotype harboring two distinct monoclonal populations. The combination of PCR and immunohistochemistry may provide indirect evidence that lymphoma is bigenotypic [5].

\section{Case Report}

A 63-year-old female patient presented to the emergency room with obstructive jaundice and dull intermittent, localized epigastric pain for 10 days, each episode lasted a few hours then resolved spontaneously, relieved by sitting forward and associated with nausea with no vomiting. She had dark urine and decreased appetite in the last 2 weeks, with unintentional weight loss. On physical examination, she was icteric with epigastric tenderness and hepatomegaly. CT scan revealed lobulated soft tissue mass lesion in close relation to the pancreatic body which can either be pancreatic in origin or an exophytic peripancreatic lymph nodal mass along with large contiguous retroperitoneal lymph nodal mass (an image of the CT is

\section{Karger'}




\section{Case Reports in Oncology}
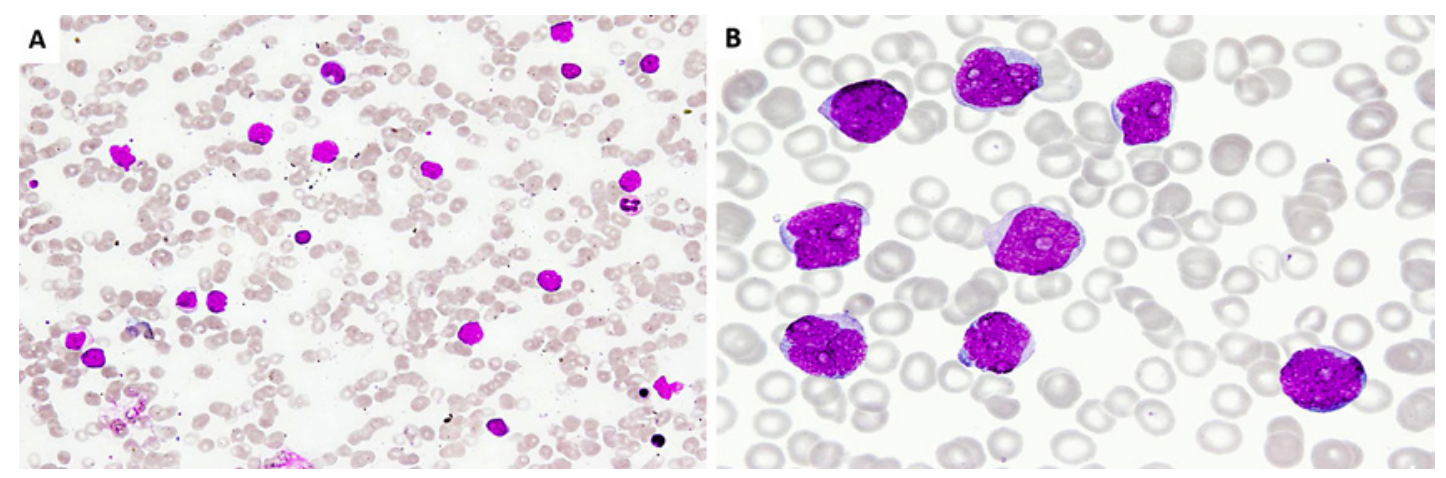

Fig. 1. A Peripheral blood smear shows leukocytosis with many immature-looking lymphoid cells (Wright stain, $\times 500$ ). B High power (composite) of many circulating immature-looking lymphoid cells, medium to large in size, with open chromatin and one or more prominent nucleoli (blast morphology) $(\times 1,000)$.

Fig. 2. Bone marrow aspirate smear shows many medium to large immature-looking cells with blast morphology (open chromatin, one or more prominent nucleoli) (Wright stain, ×1,000).



available from the author). Possibilities suggested were hematological/lymphoproliferative malignancy or pancreatic neoplasm with multiple nodal metastases. Endoscopy-guided fine needle aspiration from the pancreas revealed a few clusters of atypical cells. Her initial CBC revealed leukocytosis with $43 \times 10^{3} / \mu \mathrm{L}$ white blood cells, mild anemia with $11.2 \mathrm{~g} / \mathrm{dL}$ hemoglobin, and moderate thrombocytopenia with $88 \times 10^{3} / \mu \mathrm{L}$ platelets. Peripheral smear showed leukoerythroblastic picture, with (56\%) immature-looking atypical lymphoid cells; the majority appeared medium- to large-sized with open chromatin and one or more nucleoli (blast morphology). Some other cells appeared smaller in size with scant cytoplasm, more condensed nuclear chromatin, and inconspicuous nucleoli (shown in Fig. 1A, B). Bone marrow (BM) aspirate was infiltrated by $83 \%$ immature-looking cells having the same morphology as those seen in the peripheral blood with decreased trilineage hematopoiesis (shown in Fig. 2). BM biopsy was hypercellular for patients' age showing interstitial to diffuse infiltration by primitive-looking cells with decreased trilineage hematopoiesis. There was a focal area of tingible body macrophages with many apoptotic cells with multiple pyknotic nuclear fragments (shown in Fig. 3A, B). Immunohistochemical studies on the core biopsy showed diffuse 

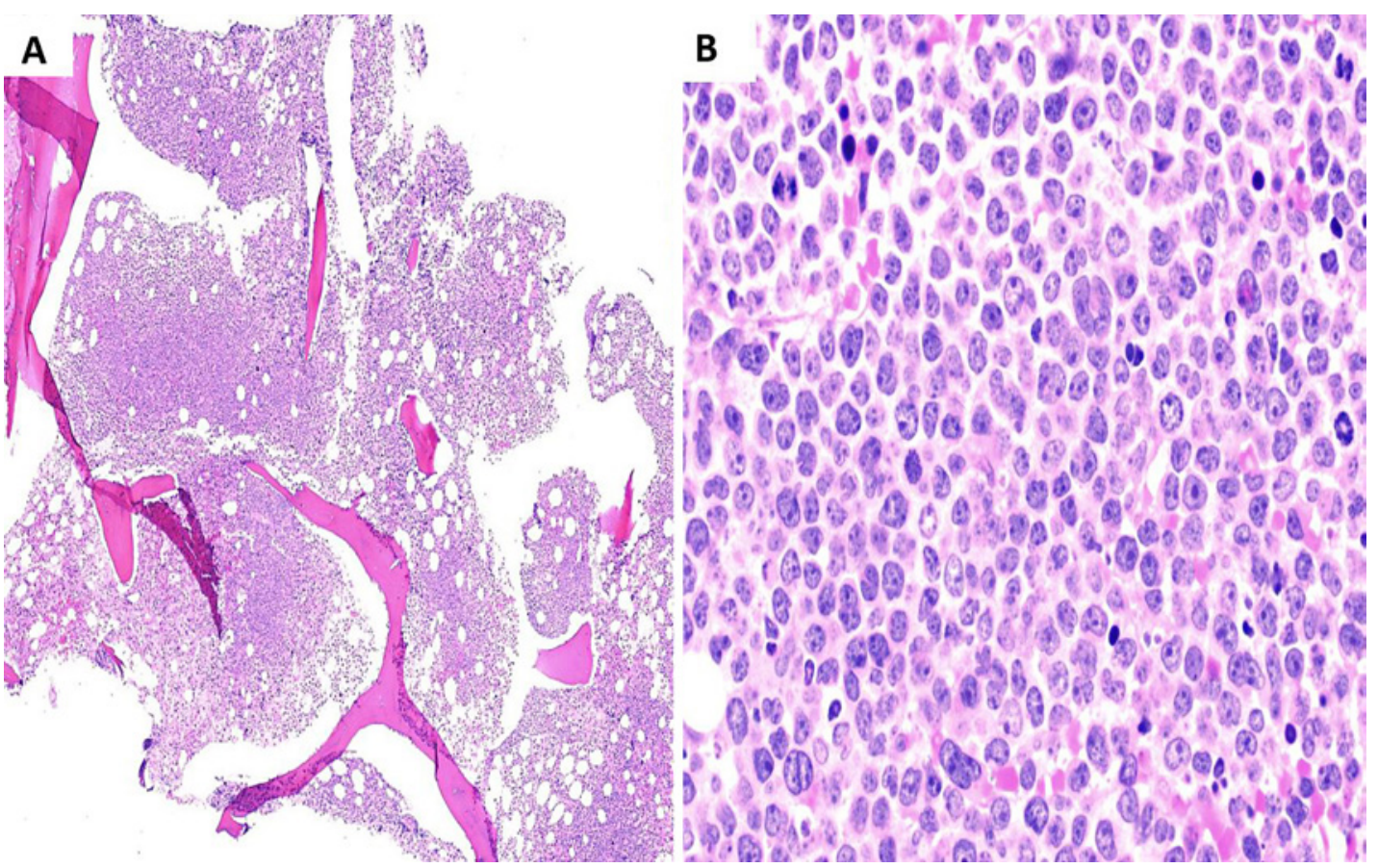

Fig. 3. Bone marrow biopsy H\&E stain A The biopsy is hypercellular for age (60\%-95\%) showing interstitial to diffuse infiltration by primitive-looking cells $(\times 100)$. B Higher power shows many medium to large-sized primitive-looking cells with primitive-looking chromatin, prominent nucleoli, and irregular/convoluted/indented nuclear borders, and relatively abundant cytoplasm with decreased trilineage hematopoiesis $(\times 500)$.

positivity for CD20, PAX5, CD79, CD10, BCL2, C-MYC (>40\%), CD3, CD4, and CD5, while negativity for CD34, CD1a, TdT, CD2, CD7, CD8, BCL6, Cyclin-D1, CD23, CD30, and ALK (shown in Fig. 4A-C).

\section{Studies for Epstein-Barr Virus}

Epstein-Barr virus (EBV) RNA detection by chromogenic in situ hybridization for EBVencoded small RNA performed on formalin-fixed, paraffin-embedded tissue was negative. The case was also negative for EBV PCR on peripheral blood.

\section{Flow Cytometry Analysis}

Flow cytometry on BM aspirate revealed an abnormal major population of cells comprising 50\% expressing CD45, CD19 (dim), CD20, cCD79a, CD22, CD10, CD4, HLADR, and cBCl2 with partial expression of CD5 (dim) and showing lambda light chain restriction. This population was positive for cCD3. The cells were negative for CD34, TdT, CD1a, cMPO, CD117, CD13, CD14, CD15, CD33, CD64, CD61, CD41, glyophorin A, CD36, CD11b, CD11c, sCD3, CD2, CD7, CD8, TCR alpha/beta, TCR gamma/delta, CD56, CD57, FMC7, CD200, CD23, sIgD, sIgA, sIgM, and sIgG. The analysis showed, also, another abnormal minor population of lambda restricted monotypic B cells comprising 8\% with dimmer CD45 (blast gate) and downregulation of CD10, showing the same immunophenotype as the major population (expressing all B-cell antigens); however, it was negative for the T-cell antigen $\mathrm{CCD} 3, \mathrm{CD} 5$, and CD4 (shown in Fig. 5). 


\section{Case Reports in Oncology}
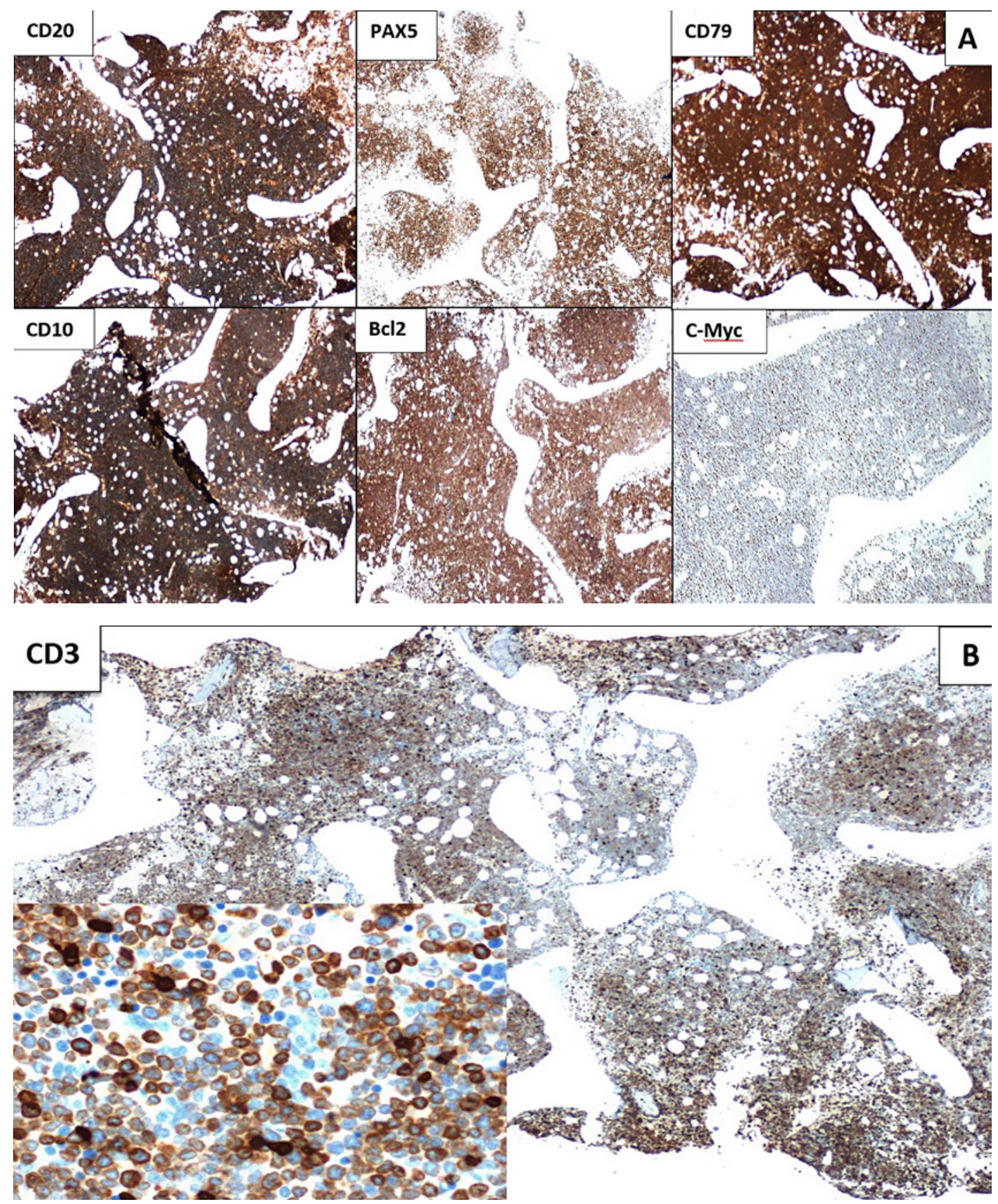

Fig. 4. IHC stain shows diffuse positivity for CD20, CD10, CD79, PAX5, BCL2, and C-MYC ( $>40 \%)(\mathbf{A} ; \times 100)$. B CD3 ( $\times 100$; inset $\times 500)$. C Diffuse positivity for CD4, CD5, and CD99. The cells are negative for CD34, TdT, and CD1a $(\times 100)$.

(Figure continued on next page.)

FISH and Cytogenetic Analysis

Conventional cytogenetic analysis revealed an abnormal female karyotype with dominant complex clone having multiple structural abnormalities including non-recurrent translocations, deletions, and duplications in $81 \%$ of cells analyzed.

Karyotype: 45,XX,add(2)(p13),add(5)(q22),?t(8;22)(q24;q11.2),add(10)(q26),-10,der (13)t(10;13)(q11.2;q14),del(13)(q14),?der(17)t(2;17)(p21;q25), add(17)(p13),?i(19)(q10) [13]/46,idem,+16[13]/46,XX[6]. Fluorescent in situ hybridization (FISH) analyses using IGH, 


\section{Case Reports in Oncology}

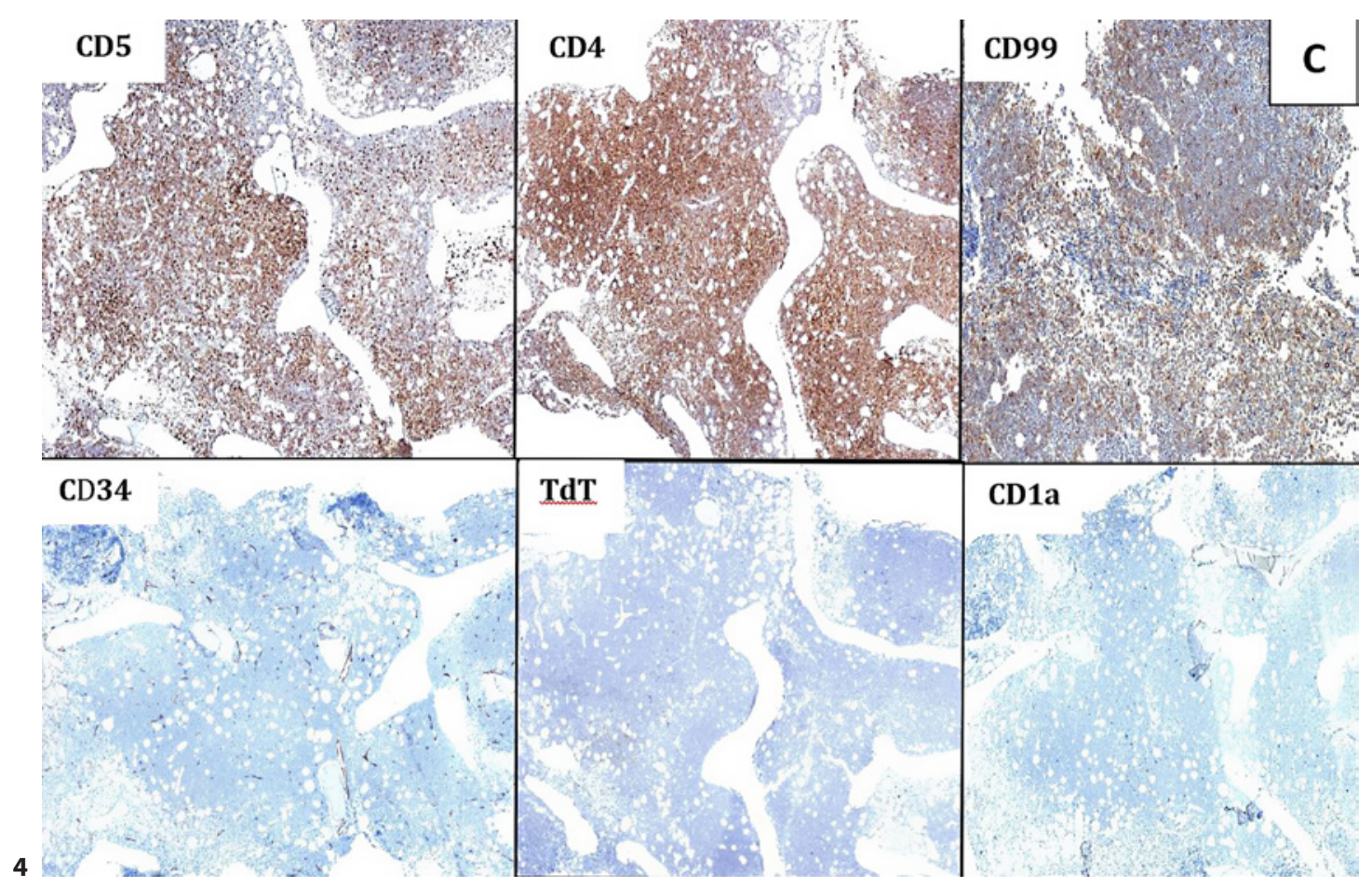

IGH/MYC/CEP 8, D7S486/CEP, TRA/D 7, MLL, CDKN2A/CEP9, and BCL6 probes were found to be normal.

Our final diagnosis was peripheral blood and BM involvement by EBV-negative highgrade lymphoid neoplasm (in leukemic phase with blast morphology) and an ambiguous immunophenotype with a differential diagnosis that may include the rare entity of bigenotypic lymphoma or an unusual case of high-grade B-cell lymphoma with aberrant expression of T-cell markers (biphenotypic lymphomas).

Molecular Analysis for IgH/TCR Clonality by PCR

PCR analysis of genomic DNA from marrow was used to identify clonal B-cell and T-cell populations using primers that target the conserved framework (FR) and joining (J) regions of the respective genes (Invivoscribe Identiclone Clonality Assay). The assay identifies immunoglobulin heavy chain (IgH) and T-cell receptor (TCR) gene rearrangements that are unique in length and sequence to provide a clonal signature of lymphoproliferative disease. Unequivocal dominant PCR products corresponding in size to those expected from clonal IGH gene rearrangements were amplified by PCR analysis (shown in Fig. 6). There was no clear-cut evidence of T-cell clonality but the TCR reactions produced dominant peaks of limited heights, within polyclonal backgrounds, considered equivocal with respect to T-cell clonality and did not exclude the possibility of a low-level T-cell clone (shown in online supplementary Fig. 1A and B; for all online suppl. material, see www. karger.com/doi/10.1159/000510403).

In conclusion, the molecular data was consistent with a clonal B-cell population and the possibility of a subclone with cross-lineage gene rearrangement or concurrent independent T-cell clones representing a minority of $\mathrm{T}$ cells could not be excluded. 

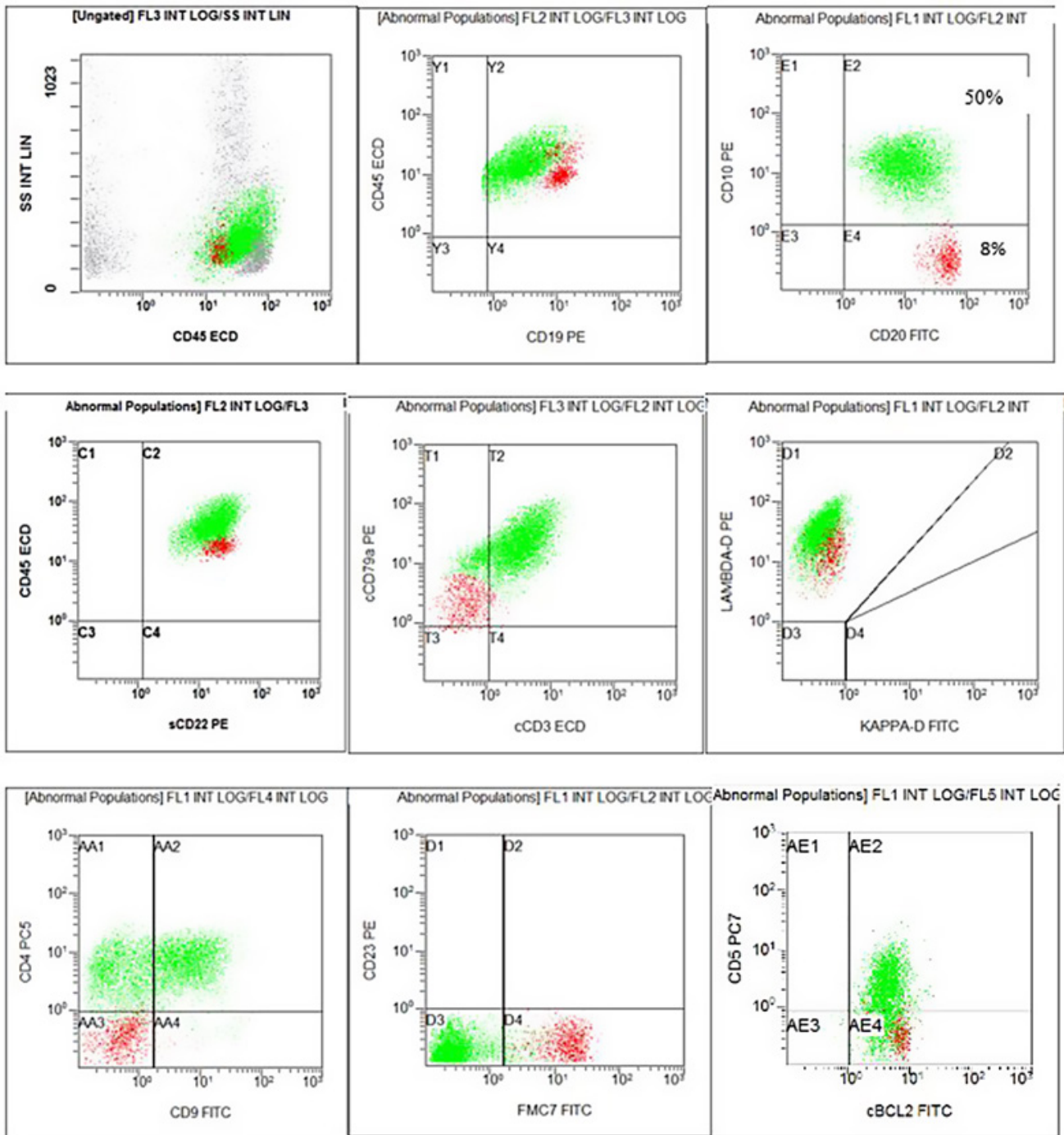

Fig. 5. Flow cytometry on bone marrow shows an abnormal population of cells comprising 50\% (green color) with moderate CD45, expressing CD19, CD20, cCD79a, CD22 CD10, CD4, HLADR, and cBCl2, with partial dim expression of CD5 and showing lambda light chain restriction. This population is positive for cCD3. Analysis shows as well an abnormal minor population of cells with dim CD45 (blast gate) comprising 8\% (red color) expressing CD19, CD20, cCD79a, CD22, cBCl2, and FMC7 and showing lambda light chain restriction. This population shows downregulation of CD10 and is negative for CCD3, CD5, and CD4.

\section{Discussion}

Non-Hodgkin lymphomas (NHL) are appointed to B-cell and T-cell lineages based on immunoprofiling and molecular studies. Herein we describe a case with an uncommon highgrade lymphoma (blast morphology) with leukemia presentation mimicking acute leukemia that was biphenotypic (expressing both T- and B-cell lineage-specific markers at the singlecell level), showing monoclonal gene rearrangement of B-cell lineage (rearrangements of the IgH region), whereas T-cell clonality by TCR gene rearrangement showed equivocal results that failed to exclude the possibility of the presence of a low-level T-cell clone. This case elab- 


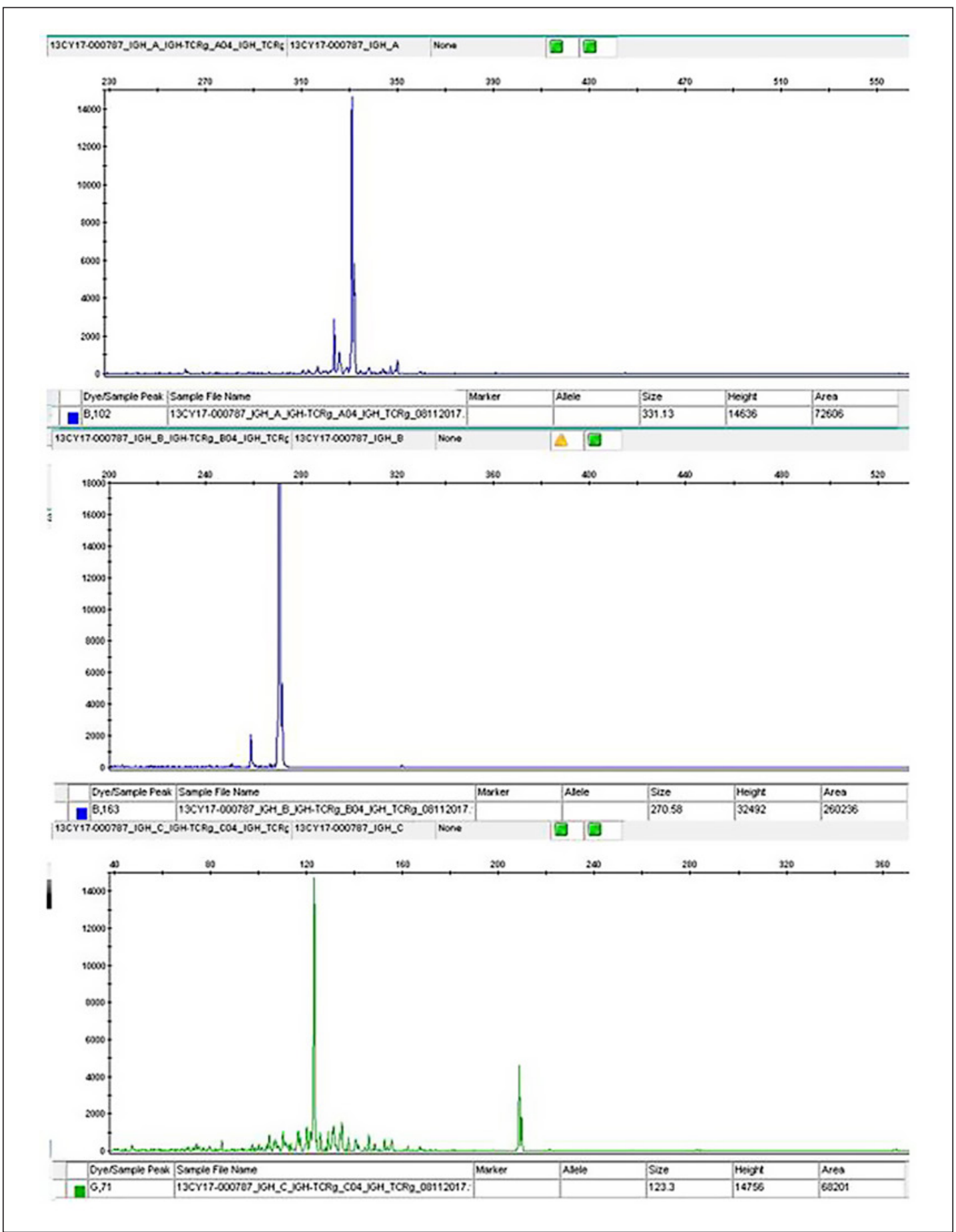

Fig. 6. Electropherograms illustrating PCR products in the expected size range for an IgH clone illustrating clonal PCR products with the FR1 (331 bp), FR2 (270 bp), and FR3 (123 bp) consistent with a clonal B-cell population.

orates how difficult it is to determine the lineage of NHL using only pan-T-cell or pan-B-cell markers. The leukemic presentation in our case, with peripheral blood and BM involvement by lymphoma cells, presenting with high total leukocytic count and blastoid morphology 
mimicking acute leukemia, imposes a challenge in the diagnosis and triggers initially using acute leukemia panel in flow cytometry immunophenotype. The analysis came to be negative for all immaturity markers (CD34, TdT, CD1a, and CD117), in addition to the moderate expression of CD45 on the major population, raising the suspicion of a mature neoplasm. A second panel including light chain assessment was performed, which revealed lambda light chain restriction. However, the co-expression of pan-B-cell-specific markers (CD19, CD20, CD22, cCD79a) as well as the most specific T-cell lineage marker (cCD3), and some T lineageassociated markers (CD4 and CD5) led to a diagnostic dilemma.

Lineage ambiguity has been documented in some hematolymphoid neoplasms, particularly in immature neoplasia, like mixed phenotype leukemia [6]. For example, a biphenotypic leukemia is defined when leukemic cells (often blasts or their equivalents) express lineagespecific antigens for one or more (commonly two) cell lineages [7]. Less commitment of the cells could explain this phenomenon once they are transformed at a progenitor stage, whereas in a more differentiated hematolymphoid neoplasm, like NH B-cell lymphoma, the lineage ambiguity is less often observed, though the aberrant expression of certain lineage-associated antigens has been well documented [8].

The CD3 molecule is expressed in the cytoplasm of prothymocytes in the cortex of the thymus and subsequently is translocated to the cell surface of common thymocytes when the cell migrates to the medulla [9]. In a mature T cell, CD3 exists as a transmembrane protein with heterodimer peptide units joined with the T-cell receptor (TCR) forming a TCR complex [10]. Due to its high degree of specificity, CD3 has been considered a specific antigen marker to define T-cell lineage in a hematolymphoid neoplasm according to the WHO classification. Expression of CD3 on mature B-cell neoplasms is exceptionally rare, with only random cases or small case series reported in the literature [11].

The detection of CD3 on a B-cell neoplasm would complicate the diagnosis and classification of the lymphoid neoplasm due to the high specificity of CD3 for T-cell lineage. This often results in an intensive study to differentiate B-cell lymphoma from T-cell lymphoma or a biphenotypic/bigenotypic B/T-cell neoplasm [12]. A distinction might become more difficult in those cases during which aberrant expression of CD3 is coupled with downregulation of B-cell antigens, like in plasmablastic lymphoma, primary effusion lymphoma, and other EBVassociated large B-cell lymphomas furthermore as those with anaplastic morphology [13]. Most of the reported cases of B- cell lymphoma with aberrant CD3 commonly express B-cellspecific antigens and this was principally seen in EBV-negative cases, expressing a full panel of B-cell antigens tested. On the other hand, in a few of the reported cases, some of the B-cell antigens were deregulated, and most of these cases were linked to EBV. In these cases, immunoglobulin heavy chain or light chain restriction $[14,15]$ helped in defining the B-cell lineage.

Wu et al. [11] also confirmed that the neoplasia can be nominated as B-cell lineage if light chain restriction is detected on the cell surface or in the cytoplasm by flow cytometry, even though when all pan-B-cell antigens are lost. Our case showed strong expression of all pan-Bcell markers and lambda light chain restriction as well indicating B-cell lineage.

It also was often reported that in B-cell lymphoma, aberrant expression of CD3 by flow cytometry is more to be cytoplasmic rather than on the surface and to be weak or focal rather than diffuse or strong on the histologic sections [11]. Our case showed a strong cytoplasmic CD3 expression by flow cytometry and diffuse strong positivity by immunohistochemistry and shows strong positivity for other T-cell-associated markers including CD4 and partial CD5. This led to a dilemma in concluding the lineage confidently and added to the diagnosis challenge whether the case represents a B-cell neoplasm with aberrant expression of T-cell markers or whether it is a real biphenotypic B/T-cell lymphoma.

A considerable number of reported cases with ambiguous immunophenotype were associated with EBV infection, supporting the hypothesis that in cases of EBV-positive DLBCL, EBV

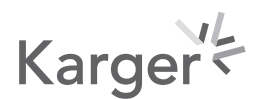


protein may stimulate lineage infidelity during malignant transformation by interfering with the expression of certain genes in the host cells and activating others, such as T-cell antigens [11]. Other hypotheses have also been anticipated, including (1) neoplastic transformation occurring at the progenitor cell level, (2) uplifting genetic repression during neoplastic transformation, and (3) neoplastic expansion of a subset B-cell population that expresses T-cell antigens [16]. In all cases of lymphoma with an ambiguous $B$ and $T$ antigen profile, it is recommended to perform EBV testing. Our case was found to be EBV negative by PCR and by EBVencoded small RNA (EBER).

An expanded literature review demounted 5 case series and 7 case reports of DLBCL with aberrant expression of CD3 with a complete set of 33 cases. Four out of 25 reported cases were double positive for both clonal IgH gene rearrangement and TCR gene rearrangement, while 4 out of 27 cases were negative for IgH gene rearrangement. The double positive cases were biphenotypic/bigenotypic lymphoid neoplasms [11]. High-throughput sequencing analysis has been widely applied to the analysis of tumor genomes, including analysis of immunoglobulin genes and TCR gene $[17,18]$. With the application of this technique combined with flow cytometry cell sorting, genotypic profiling of lymphoma with ambiguous lineage is likely feasible. In our case, molecular analysis for immunoglobulin genes confirmed the B-cell clonality, while TCR rearrangement came to be equivocal, which could not entirely exclude the possibility of a T-cell clone within the B-cell clone (bigenotypic).

It is suggested that the PAX5 molecule plays a vital role within the development of lymphocytes, arranging the maturation of lymphocytes committed to the B-cell lineage. It has been suggested that PAX5 may activate regulatory genes specific for B-cell development and repress the genes specific for $\mathrm{T}$ cells during the development of lymphocytes. With an in vitro study, Cobaleda et al. [19] demonstrated that knockout of the PAX5 gene made mature B-cells transform into other lineages, such as T cells. However, this does not explain the clinical case described herein, since there was a strong expression of PAX5.

Unfortunately, our patient died 2 days after BM examination before receiving any kind of treatment and this precludes further investigation or evaluating treatment response.

\section{Conclusion}

Discrimination between B-cell and T-cell neoplasms is clinically essential because of the variance in therapeutic management and prognosis. The current case spotlights the diagnostic challenges associated with lineage ambiguity in rare high-grade B-cell neoplasms and further emphasizes the implementation of flow cytometry immunophenotyping/immunohistochemical panels and genotypic/molecular tests for a conclusive diagnosis and classification. We also support testing EBV in high-grade lymphoid neoplasms with ambiguous lineage.

This case acts as an instructive note of the diagnostic pitfalls involved in the lineage determination of NHL using only pan-B- and pan-T-cell markers. For full interpretation and accurate diagnosis, more extensive immunoassays and molecular studies are required.

\section{Acknowledgement}

The authors would like to acknowledge co-workers and Qatar National Library, without whom this work would not have been possible.

\section{Karger'}




\section{Case Reports in Oncology}

\begin{tabular}{l|l}
\hline Case Rep Oncol 2020;13:1215-1226 \\
\hline DOI: 10.1159/000510403 & $\begin{array}{l}\text { ○ 2020 The Author(s). Published by S. Karger AG, Basel } \\
\text { www.karger.com/cro }\end{array}$ \\
\hline
\end{tabular}

Kohla et al.: High-Grade Biphenotypic Lymphoma

\section{Statement of Ethics}

The authors have no ethical conflicts to disclose. The case report was conducted ethically in accordance with the guideline of the Medical Research Center of Hamad Medical Corporation, and after obtaining their approval. Informed consent was provided by the relative of the patient to publish the case (including publication of images).

\section{Conflict of Interest Statement}

The authors declare that they have no relevant financial interests.

\section{Funding Sources}

Qatar National Library funded the study.

\section{Author Contributions}

Dr. Samah Kohla did the literature review and wrote the hematology part of the manuscript. Susanna Akiki wrote the cytogenetic/molecular part of the manuscript. Dr. Deena Mudawi, and Dr. Mohamed A. Yassin wrote the clinical part of the manuscript. Dr. Feryal A. Ibrahim, Dr. Ahmad Al-Sabbagh and Dr. Dina Soliman reviewed the hematology part of the manuscript, Dr. Reda Youssef reviewed the manuscript.

\section{References}

1 Lee M, Cha HJ, Yoon DH, Suh C, and Huh J. EBV-positive diffuse large B-cell lymphoma of the elderly with aberrant expression of CD3 and TIA-1. Blood Res. 2013 Jun;48(2):156-60.

2 Wang J, Chen C, Lau S, Raghavan RI, Rowsell EH, Said J, et al. CD3-positive large B-cell lymphoma. Am J Surg Pathol. 2009;33(4):505-12.

3 Vergier B, Dubus P, Kutschmar A, Parrens M, Ferrer J, de Mascarel A, et al. Combined analysis of T cell receptor gamma and immunoglobulin heavy chain gene rearrangements at the single-cell level in lymphomas with dual genotype. J Pathol. 2002;198(2):171-80.

4 Evans PA, Pott C, Groenen PJ, Salles G, Davi F, Berger F, et al. Significantly improved PCR-based clonality testing in B-cell malignancies by use of multiple immunoglobulin gene targets. Report of the BIOMED-2 Concerted Action BHM4-CT98-3936. Leukemia. 2007;21(2):207-14.

5 Hansson M, Jerkeman M, Dictor M. Biphenotypic bigenotypic lymphoma with simultaneous expression of PAX5/BSAP and B- and T-cell markers. Eur J Haematol. 2007;79(2):159-65.

6 Lau LG, Tan LK, Koay ES, Ee MH, Tan SH, Liu TC. Acute lymphoblastic leukemia with the phenotype of a putative B-cell/T-cell bipotential precursor. Am J Hematol. 2004;77(2):156-60.

7 Matutes E, Morilla R, Farahat N, Carbonell F, Swansbury J, Dyer M, et al. Definition of acute biphenotypic leukemia. Haematologica. 1997;82(1):64-6.

8 Mitrovic Z, Ilic I, Nola M, Aurer I, Sonicki Z, Basic-Kinda S, et al. CD43 expression is an adverse prognostic factor in diffuse large B-cell lymphoma. Clin Lymphoma Myeloma. 2009;9(2):133-7.

9 Harris NL, Jaffe ES, Diebold J, Flandrin G, Muller-Hermelink HK, Vardiman J, et al. The World Health Organization classification of hematological malignancies report of the Clinical Advisory Committee Meeting, Airlie House, Virginia, November 1997. Mod Pathol. 2000;13(2):193-207.

10 Clevers H, Alarcon B, Wileman T, Terhorst C. The T cell receptor/CD3 complex: a dynamic protein ensemble. Annu Rev Immunol. 1988;6:629-62.

11 Wu B, Vallangeon B, Galeotti J, Sebastian S, Rehder C, Wang E. Epstein-Barr virus-negative diffuse large B cell lymphoma with aberrant expression of CD3 and other T cell-associated antigens: report of three cases with a review of the literature. Ann Hematol. 2016;95(10):1671-83.

12 Hansson M, Jerkeman M, Dictor M. Biphenotypic bigenotypic lymphoma with simultaneous expression of PAX5/BSAP and B- and T-cell markers. Eur J Haematol. 2007;79(2):159-65. 
13 Oliveira JL, Grogg KL, Macon WR, Dogan A, Feldman AL. Clinicopathologic features of B-Cell lineage neoplasms with aberrant expression of CD3: a study of 21 cases. Am J Surg Pathol. 2012;36(9):1364-70.

14 Sun J, Medeiros LJ, Lin P, Lu G, Bueso-Ramos CE, You MJ. Plasmablastic lymphoma involving the penis: a previously unreported location of a case with aberrant CD3 expression. Pathology. 2011;43(1):54-7.

15 Stein H, Harris NL, Campo E. Plasmablastic lymphoma. In: Swerdllow S, Campo E, Harris NL (eds). WHO Classification of Tumours of Haematopoietic and Lymphoid Tissues, 4th ed. IARC Press, France, 2008, pp 256-7.

16 Petitjean B, Jardin F, Joly B, Martin-Garcia N, Tilly H, Picquenot JM, et al. Pyothorax-associated lymphoma: a peculiar clinicopathologic entity derived from B cells at late stage of differentiation and with occasional aberrant dual B- and T-cell phenotype. Am J Surg Pathol. 2002;26(6):724-32.

17 Carlotti E, Wrench D, Rosignoli G, Marzec J, Sangaralingam A, Hazanov L, et al. High Throughput Sequencing Analysis of the Immunoglobulin Heavy Chain Gene from Flow-Sorted B Cell Sub-Populations Define the Dynamics of Follicular Lymphoma Clonal Evolution. PLoS One. 2015;10(9):e0134833.

18 Miyai M, Eikawa S, Hosoi A, Iino T, Matsushita H, Isobe M, et al. Detection and Tracking of NY-ESO-1-Specific CD8+ T Cells by High-Throughput T Cell Receptor $\beta$ (TCRB) Gene Rearrangements Sequencing in a PeptideVaccinated Patient. PLoS One. 2015;10(8):e0136086

19 Cobaleda C, Jochum W, Busslinger M. Conversion of mature B cells into T cells by dedifferentiation to uncommitted progenitors. Nature. 2007;449(7161):473-7. 